\title{
Correction to: Regioselective synthesis of pyridines by redox alkylation of pyridine $\mathrm{N}$-oxides with malonates
}

\author{
Miran Lemmerer ${ }^{1} \cdot$ Christopher J. Teskey $^{1} \cdot$ Daniel Kaiser $^{1} \cdot$ Nuno Maulide $^{1}$ (])
}

Published online: 23 January 2018

(C) Springer-Verlag GmbH Austria, part of Springer Nature 2018

\section{Correction to: Monatsh Chem} https://doi.org/10.1007/s00706-017-2081-y

The original version of this article unfortunately contained mistakes. In section "General procedure" some numbers were missing. The corrected text is given below.

\section{General procedure}

All flasks and stirrer bars were flame dried before use. To the $N$-oxide ( $0.2 \mathrm{mmol}, 1.0$ equiv.), dissolved in $2 \mathrm{~cm}^{3}$ dichloromethane was added $\mathrm{Tf}_{2} \mathrm{O}(0.3 \mathrm{mmol}, 1.5$ equiv. $)$ at $0{ }^{\circ} \mathrm{C}$. In another flask, a suspension of $\mathrm{NaH}(0.7 \mathrm{mmol}, 3.5$ equiv.) in $1 \mathrm{~cm}^{3}$ tetrahydrofuran was cooled to $0{ }^{\circ} \mathrm{C}$ and the malonate $(0.7 \mathrm{mmol}, 3.5$ equiv. $)$ was added. After $15 \mathrm{~min}$, the malonate solution was added to the activated $\mathrm{N}$-oxide solution and the mixture was stirred at room temperature for $1 \mathrm{~h}$. The reaction was quenched with $\mathrm{NH}_{4} \mathrm{Cl}$ solution and the aqueous phase was extracted with dichloromethane. The combined organic layers were washed with brine before being dried over $\mathrm{MgSO}_{4}$. The solvents were removed under reduced pressure and the crude product was purified by column chromatography.

Dibenzyl 2-(2,6-dimethylpyridin-4-yl)malonate (6a, $\mathrm{C} 24 \mathrm{H} 23 \mathrm{NO} 4)$ The product was prepared according to the general procedure. Purification by column chromatography (EtOAc:heptane $=1: 1)$ yielded the product $(41.0 \mathrm{mg}$,

The original article can be found online at https:// doi.org/10.1007/s00706-017-2081-y.

Nuno Maulide

nuno.maulide@univie.ac.at

1 Institute of Organic Chemistry, University of Vienna, Währinger Strasse 38, 1090 Vienna, Austria
$53 \%)$ as a pale yellow solid. ${ }^{1} \mathrm{H}$ NMR $\left(400 \mathrm{MHz}, \mathrm{CDCl}_{3}\right)$ : $\delta=7.28-7.20(\mathrm{~m}, 10 \mathrm{H}), 6.88(\mathrm{~s}, 2 \mathrm{H}), 5.11(\mathrm{dd}, J=12.0$, $18.1 \mathrm{~Hz}, 4 \mathrm{H}), 4.56(\mathrm{~s}, 1 \mathrm{H}), 2.43(\mathrm{~s}, 6 \mathrm{H}) \mathrm{ppm} ;{ }^{13} \mathrm{C} \mathrm{NMR}$ $\left(101 \mathrm{MHz}, \mathrm{CDCl}_{3}\right): \delta=167.0,158.4,141.5,135.1,128.7$, 128.7, 128.4, 120.9, 67.9, 57.3, 24.6 ppm; IR: $\bar{v}=3064$, $3033,2955,2922,1732,1605,1569,1497,1453,1375$, 1297, $1140 \mathrm{~cm}^{-1}$; HRMS (ESI): $\mathrm{m} / \mathrm{z}$ calculated for $[\mathrm{M}+\mathrm{H}]^{+}$390.1700, found 390.1701.

Diethyl 2-(2,6-dimethylpyridin-4-yl)-2-fluoromalonate (6b, $\mathrm{C}_{14} \mathrm{H}_{18} \mathrm{FNO}_{4}$ ) The product was prepared according to the general procedure. Purification by column chromatography (EtOAc:heptane $=1: 3)$ yielded the product $(34.3 \mathrm{mg}$, $61 \%)$ as a pale yellow liquid. ${ }^{1} \mathrm{H}$ NMR $\left(400 \mathrm{MHz}, \mathrm{CDCl}_{3}\right)$ : $\delta=7.19(\mathrm{~s}, 2 \mathrm{H}), 4.33(\mathrm{q}, J=7.1,4 \mathrm{H}), 2.56(\mathrm{~s}, 6 \mathrm{H}), 1.32$ $(\mathrm{t}, J=7.1 \mathrm{~Hz}, 6 \mathrm{H}) \mathrm{ppm} ;{ }^{13} \mathrm{C}$ NMR $\left(101 \mathrm{MHz}, \mathrm{CDCl}_{3}\right)$ : $\delta=164.9 \quad(\mathrm{~d}, \quad J=25.0 \mathrm{~Hz}), \quad 158.3, \quad 142.4 \quad(\mathrm{~d}$, $J=22.4 \mathrm{~Hz}), \quad 116.8 \quad(\mathrm{~d}, \quad J=9.0 \mathrm{~Hz}), \quad 93.2 \quad(\mathrm{~d}$, $J=202.9 \mathrm{~Hz}), 63.4,24.8,14.0 \mathrm{ppm} ;{ }^{19} \mathrm{~F}$ NMR $(659 \mathrm{MHz}$, $\left.\mathrm{CDCl}_{3}\right):-165.2 \mathrm{ppm}$; IR: $\bar{v}=2983,2927,1753,1604$, $1569,1445,1412,1369,1270,1230,1174,1105,1044$, $1010 \mathrm{~cm}^{-1}$; HRMS (ESI): $\mathrm{m} / \mathrm{z}$ calculated for $[\mathrm{M}+\mathrm{H}]^{+}$ 284.1293, found 284.1292.

Diethyl 2-(2-cyanoethyl)-2-(2,6-dimethylpyridin-4-yl)malonate $\left(6 \mathbf{c}, \mathrm{C}_{17} \mathrm{H}_{22} \mathrm{~N}_{2} \mathrm{O}_{4}\right)$ The product was prepared according to the general procedure. Purification by column chromatography (EtOAc:heptane $=1: 1)$ yielded the product $(48.4 \mathrm{mg}, 76 \%)$ as a pink liquid. ${ }^{1} \mathrm{H}$ NMR $(400 \mathrm{MHz}$, $\left.\mathrm{CDCl}_{3}\right): \delta=6.90(\mathrm{~s}, 2 \mathrm{H}), 4.32-4.24(\mathrm{~m}, 4 \mathrm{H}), 2.61-2.57(\mathrm{~m}$, $2 \mathrm{H}), 2.54(\mathrm{~s}, 6 \mathrm{H}), 2.37-2.33(\mathrm{~m}, 2 \mathrm{H}), 1.28(\mathrm{t}, J=7.1 \mathrm{~Hz}$, $6 \mathrm{H}) \mathrm{ppm} ;{ }^{13} \mathrm{C} \mathrm{NMR}\left(125 \mathrm{MHz}, \mathrm{CDCl}_{3}\right): \delta=168.8,158.6$, 145.0, 119.0, 118.9, 62.6, 61.3, 32.0, 24.8, 14.0, 13.5 ppm; IR: $\bar{v}=2982,2937,2249,1728,1603,1564,1445,1368$, 1254, 1188, 1079, $1016 \mathrm{~cm}^{-1}$; HRMS (ESI): $\mathrm{m} / z$ calculated for $[\mathrm{M}+\mathrm{H}]^{+}$319.1652, found 319.1651. 
Diethyl 2-(2,6-dimethylpyridin-4-yl)-2-methylmalonate (6d, $\mathrm{C}_{15} \mathrm{H}_{21} \mathrm{NO}_{4}$ ) The product was prepared according to the general procedure. Purification by column chromatography $($ EtOAc:heptane $=1: 1)$ yielded the product (33.6 mg, 60\%) as a pale yellow liquid. ${ }^{1} \mathrm{H}$ NMR $\left(400 \mathrm{MHz}, \mathrm{CDCl}_{3}\right): \delta=6.94(\mathrm{~s}, 2 \mathrm{H}), 4.25(\mathrm{~m}, 4 \mathrm{H}), 2.52$ $(\mathrm{s}, 6 \mathrm{H}), 1.81(\mathrm{~s}, 3 \mathrm{H}), 1.26(\mathrm{t}, J=7.2 \mathrm{~Hz}, 6 \mathrm{H}) \mathrm{ppm} ;{ }^{13} \mathrm{C}$ NMR (101 MHz, $\left.\mathrm{CDCl}_{3}\right): \delta=170.7,157.9,147.8,119.1$, 62.1, 58.6, 24.8, 22.2, $14.1 \mathrm{ppm}$; IR: $\bar{v}=2982,1728$, 1604, 1564, 1447, 1414, 1377, 1253, 1181, 1105, $1017 \mathrm{~cm}^{-1}$; HRMS (ESI): $\mathrm{m} / z$ calculated for $[\mathrm{M}+\mathrm{H}]^{+}$ 280.1543 , found 280.1543 .

Diethyl 2-allyl-2-(2,6-dimethylpyridin-4-yl)malonate (7a, $\mathrm{C}_{17} \mathrm{H}_{23} \mathrm{NO}_{4}$ ) The product was prepared according to the general procedure. Purification by column chromatography (EtOAc:heptane $=1: 3$ ) yielded the product (43.6 mg, $71 \%$ ) as a pale yellow liquid. ${ }^{1} \mathrm{H} \mathrm{NMR}\left(400 \mathrm{MHz}, \mathrm{CDCl}_{3}\right)$ : $\delta=7.01(\mathrm{~s}, 2 \mathrm{H}), 5.75-5.64(\mathrm{~m}, 1 \mathrm{H}), 5.08(\mathrm{~m}, 1 \mathrm{H}), 5.04(\mathrm{~s}$, $1 \mathrm{H}), 4.29-4.16(\mathrm{~m}, 4 \mathrm{H}), 3.00(\mathrm{~d}, J=7.1 \mathrm{~Hz}, 2 \mathrm{H}), 2.52$ (s, $6 \mathrm{H}), 1.25(\mathrm{t}, J=7.1,6 \mathrm{H}) \mathrm{ppm} ;{ }^{13} \mathrm{C} \mathrm{NMR}(101 \mathrm{MHz}$, $\left.\mathrm{CDCl}_{3}\right): \delta=169.5,157.8,146.3,132.5,119.7,119.4$, $62.4,62.0,40.3,24.8,14.1 \mathrm{ppm} ; \mathrm{IR}: \bar{v}=2981,2926$, 1729, 1602, 1563, 1443, 1414, 1367, 1295, 1270, 1230, 1196, $1162 \mathrm{~cm}^{-1}$; HRMS (ESI): $\mathrm{m} / z$ calculated for $[\mathrm{M}+\mathrm{H}]^{+}$306.1700, found 306.1703.

Diethyl 2-allyl-2-(4-methylpyridin-2-yl)malonate (7b, $\mathrm{C}_{16-}$ $\mathrm{H}_{21} \mathrm{NO}_{4}$ ) The product was prepared according to the general procedure. Purification by column chromatography (EtOAc:heptane $=1: 10)$ yielded the product $(48.8 \mathrm{mg}$, $84 \%$ ) as a pale yellow liquid. ${ }^{1} \mathrm{H}$ NMR (400 $\mathrm{MHz}, \mathrm{CDCl}_{3}$ ): $\delta=8.39 \quad(\mathrm{dd}, \quad J=0.5, \quad 5.0 \mathrm{~Hz}, 1 \mathrm{H}), 7.56 \quad($ app $\mathrm{t}$, $J=0.7 \mathrm{~Hz}, 1 \mathrm{H}), 7.01-6.99(\mathrm{~m}, 1 \mathrm{H}), 5.82-5.75(\mathrm{~m}, 1 \mathrm{H})$, 5.04-4.99 (m, 2H), 4.27-4.20 (m, 4H), $3.12(\mathrm{~d}$, $J=7.2 \mathrm{~Hz}, 2 \mathrm{H}), 2.36(\mathrm{~s}, 3 \mathrm{H}), 1.24(\mathrm{t}, J=7.1 \mathrm{~Hz}, 6 \mathrm{H})$ ppm; ${ }^{13} \mathrm{C} \mathrm{NMR}\left(101 \mathrm{MHz}, \mathrm{CDCl}_{3}\right): \delta=169.9,156.6$,
148.6, 147.1, 133.6, 124.8, 123.5, 118.6, 65.3, 61.7, 40.4, 21.4, 14.1 ppm; IR: $\bar{v}=2980,2936,1729,1601,1444$, 1298, $1195 \mathrm{~cm}^{-1}$; HRMS (ESI): $\mathrm{m} / \mathrm{z}$ calculated for $[\mathrm{M}+\mathrm{H}]^{+}$292.1543, found 292.1543.

Diethyl 2-allyl-2-(4-phenylpyridin-2-yl)malonate (7c, $\mathrm{C}_{21-}$ $\mathrm{H}_{23} \mathrm{NO}_{4}$ ) The product was prepared according to the general procedure. Purification by column chromatography (EtOAc:heptane = 1:10) yielded the product $(48.0 \mathrm{mg}$, $68 \%$ ) as a pale yellow liquid. ${ }^{1} \mathrm{H}$ NMR (400 $\left.\mathrm{MHz}, \mathrm{CDCl}_{3}\right)$ : $\delta=8.59(\mathrm{dd}, J=0.7,5.1 \mathrm{~Hz}, 1 \mathrm{H}), 7.88(\mathrm{dd}, J=0.7$, $1.7 \mathrm{~Hz}, 1 \mathrm{H}), 7.65-7.63(\mathrm{~m}, 2 \mathrm{H}), 7.48-7.41(\mathrm{~m}, 4 \mathrm{H})$, 5.86-5.79 (m, 1H), 5.06-5.02 (m, 2H), 4.30-4.24 (m, 4H), $3.17(\mathrm{~d}, J=7.1 \mathrm{~Hz}, 2 \mathrm{H}), 1.26(\mathrm{t}, J=7.1 \mathrm{~Hz}, 6 \mathrm{H}) \mathrm{ppm}$; ${ }^{13} \mathrm{C}$ NMR $\left(125 \mathrm{MHz}, \mathrm{CDCl}_{3}\right): \delta=169.8,157.2,149.2$, $148.5,138.6,133.3,129.2,129.1,127.3,122.4,120.7$, $119.0,65.5,61.8,40.5,14.2 \mathrm{ppm}$; IR: $\bar{v}=3062,2980$, 2935, 1729, 1594, 1547, 1467, 1225, $1036 \mathrm{~cm}^{-1}$; HRMS (ESI): $m / z$ calculated for $[\mathrm{M}+\mathrm{H}]^{+} 354.1700$, found 354.1699 .

Diethyl 2-allyl-2-(4-cyanopyridin-2-yl)malonate (7d, $\mathrm{C}_{16}$ $\mathrm{H}_{18} \mathrm{~N}_{2} \mathrm{O}_{4}$ ) The product was prepared according to the general procedure. Purification by column chromatography (EtOAc:heptane = 1:10) yielded the product $(10.3 \mathrm{mg}$, $17 \%)$ as a pale yellow liquid. ${ }^{1} \mathrm{H} \mathrm{NMR}\left(400 \mathrm{MHz}, \mathrm{CDCl}_{3}\right)$ : $\delta=8.72(\mathrm{dd}, \quad J=0.9, \quad 5.0 \mathrm{~Hz}, 1 \mathrm{H}), 7.98 \quad($ app t, $J=1.3 \mathrm{~Hz}, 1 \mathrm{H}), 7.43(\mathrm{dd}, J=1.3,5.0 \mathrm{~Hz}, 1 \mathrm{H}), 5.74-5.63$ $(\mathrm{m}, 1 \mathrm{H}), 5.04-5.01(\mathrm{~m}, 2 \mathrm{H}), 4.29-4.22(\mathrm{~m}, 4 \mathrm{H}), 3.12(\mathrm{~d}$, $J=7.3 \mathrm{~Hz}, 2 \mathrm{H}), 1.25(\mathrm{t}, J=7.1 \mathrm{~Hz}, 6 \mathrm{H}) \mathrm{ppm} ;{ }^{13} \mathrm{C} \mathrm{NMR}$ $\left(101 \mathrm{MHz} \mathrm{CDCl}_{3}\right): \delta=169.0,158.5,149.6,132.4,126.5$, 124.0, 120.5, 119.7, 116.8, 65.3, 62.2, 40.3, 14.1 ppm; IR: $\bar{v}=3077,2981,2933,2239$, 1730, 1594, 1467, 1299, 1168, $1044 \mathrm{~cm}^{-1}$; HRMS (ESI): $\mathrm{m} / \mathrm{z}$ calculated for $[\mathrm{M}+\mathrm{Na}]^{+}$325.1159, found 325.1157 .

The original article has been corrected. 